\title{
Optimizing Longitudinal Amyloid- $\beta$ PET Measurement: The Challenges of Intensity Normalization
}

\author{
Susan M. Landau \\ Helen Wills Neuroscience Institute, University of California Berkeley, Berkeley, California; and Molecular Biophysics and Integrated \\ Bioimaging, Lawrence Berkeley National Laboratory, Berkeley, California
}

See the associated article on page 1583.

A ccurate measurement of amyloid- $\beta$ (A $\beta$ ) PET tracer retention has become an increasingly important feature of neuroimaging research in Alzheimer disease (AD). Although initial A $\beta$ PET work mostly focused on abnormal/normal ( $+/-$ ) categorization, the field has moved toward more precise measurement techniques in order to detect $A \beta$ at and even below the positivity threshold in hopes of understanding the initial stages of pathologic change as well as points of potential effective intervention. Clinical trials such as A4 using PET to screen for A $\beta$ positivity rely on the definition of an SUV ratio (SUVR) threshold or threshold range that can identify those who are early enough in the course of disease to still benefit from an $\mathrm{A} \beta$-targeting therapy (1). In addition, recent research has emphasized the earliest detection of A $\beta$ pathology, how it changes over time, and how it relates to neurodegenerative and cognitive changes (2-6), all of which depend on the accurate and precise measurement of $\mathrm{A} \beta$ on a continuous scale.

Although the selection of a reference region may appear to be a technical detail in PET image analysis, it has a major influence on A $\beta$ PET SUVRs. Cross-sectional A $\beta$ PET analyses have frequently used the cerebellar cortex or the whole cerebellum (white plus gray matter) because these regions are known to be relatively free of $A \beta$ in AD. Few studies have compared PET SUVRs calculated using different regions with one another, but existing work suggests that different reference regions are far from interchangeable $(7,8)$. For example, longitudinal florbetapir PET cortical SUVRs calculated using a whole cerebellum reference region are only minimally predictive of the same cortical change calculated using a white matter (WM) reference region (9). This reinforces the idea that reference region selection is a methodologic decision worthy of careful consideration since it is likely to significantly influence study findings.

For longitudinal studies in particular, in which accumulation may be less than $1 \%$ per year, the reference region that is selected may determine whether any change is detected at all. Several studies have reported that longitudinal florbetapir PET (9-11) and Pittsburgh compound B (PiB) PET (12) SUVRs are more stable

Received Sep. 7, 2018; revision accepted Sep. 10, 2018.

For correspondence or reprints contact: Susan M. Landau, 118 Barker Hall MC \#3190, University of California Berkeley, Berkeley, CA 94720-3190.

E-mail: slandau@berkeley.edu

Published online Sep. 13, 2018.

COPYRIGHT @ 2018 by the Society of Nuclear Medicine and Molecular Imaging. DOI: 10.2967/jnumed.118.212662 and more plausible (e.g., roughly linear, nondecreasing) when the reference region contains WM than when it does not (e.g., whole cerebellum alone). WM may result in more stable signal because it is larger, less influenced by registration problems or poor regional definition or segmentation, and less vulnerable to problems with scatter correction related to proximity to the bottom of the field of view.

On the other hand, WM signal is poorly understood, complicating its utility as a reference region. Most $A \beta$ pathology in $A D$ is located in the cortex, but $A \beta$ pathology has also been observed in WM $(13,14)$. In A $\beta$ PET imaging, tracer retention in WM is frequently as high as in the cortex and is considered nonspecific. This WM retention may be explained by slower tracer clearance compared with gray matter (15), tracer lipophilicity (16), and the $\beta$-pleated sheet structure of myelin-binding protein (17). However, $\mathrm{PiB}$ retention is also reduced in WM lesions $(18,19)$. Importantly, these factors are ligand-specific and could account for differing proportions of WM signal depending on which ligand is used. Furthermore, isolating WM from cortical signal is challenging because of the low spatial resolution of PET; elevated signal in the cortex can influence WM signal and vice versa.

Selection of an optimal reference region is further complicated by the lack of a gold standard for longitudinal measurement. Nonetheless, reference region stability over time and over the course of disease is a critical feature. In this issue of The Journal of Nuclear Medicine, Lowe et al. perform an unusually comprehensive set of PiB PET analyses to determine whether WM is an acceptable reference region for measuring cortical $\mathrm{PiB}$ retention throughout middle to older age and at different degrees of $A \beta$ burden (20). The authors use both cross-sectional and longitudinal analyses across the spectrum of disease to show that WM retention (but not cerebellar crus or whole cerebellum retention) increases with age and at moderate levels of cortical A $\beta$ (SUVR of about 1.9-2.1).

The authors performed several critical analyses to examine cortical and WM regions separately and minimize signal contamination that would complicate interpretation of WM signal. First, they performed 2-compartment partial-volume correction, which adjusts for the influence of cerebral spinal fluid on neighboring WM or gray matter but not for the influence of WM on gray matter or vice versa. To address the latter issue, the authors examined periventricular WM separately from the rest of subcortical WM, because periventricular regions are far enough away from the cortex to eliminate the influence of cortex on WM via partialvolume effects. Compared with subcortical WM that neighbors cortex, periventricular WM regions were less elevated with older age and at moderate levels of cortical $\mathrm{A} \beta$, supporting the assumption 
that rising cortical $A \beta$ contributes to the observed WM signal elevations. However, importantly, elevations in periventricular WM were still significant, so these elevations seem to be independent of cortical signal. Finally, they examined WM SUVs (using participant weight and injected dose) to verify that the SUVR increases they observed in WM (in this case, as the SUVR numerator or target region) were not driven by reductions in cerebellar gray matter (the SUVR denominator).

There are a several important implications of these results. First, whereas the study cannot determine the neurochemical basis of WM binding, the pattern of findings is consistent with the possibility that slower clearance in WM accounts for increased WM signal, because this would be expected to worsen with age. On the other hand, the findings argue against the idea that reduced signal associated with WM lesions (such as WM hyperintensities) have a major influence on WM retention overall, because we would expect to see age-related reductions in WM retention accompanying agerelated increases in WM hyperintensities, instead of the increasing, age-related WM retention that was observed. More importantly, the findings indicate that instability of WM over time could compromise longitudinal cortical PiB measurements when a WM reference region is used, perhaps underestimating cortical increases at older ages and for those with moderate $A \beta$ burden. On the other hand, the authors acknowledge that problems in cortical longitudinal PiB SUVR measurement due to WM instability are likely to be minimal for studies lasting just a few years (which is most A $\beta$ PET studies). For these shorter studies, the reduction in variability resulting from using a larger WM reference region might exceed the drawback of the WM instability.

Examining the behavior of WM binding with other ligands and in other samples will be critical for continuing to optimize longitudinal PET measurement in future AD research. This work will also benefit clinical trials screening for the presence of $A \beta$ and tau and tracking longitudinal changes in this pathology over time. A $\beta$ PET has had a head start in defining tools and strategies for accurate measurement that may also eventually benefit tau PET despite the initial challenges of greater regional specificity and complex patterns of off-target binding (21).

\section{REFERENCES}

1. Sperling RA, Rentz DM, Johnson KA, et al. The A4 study: stopping AD before symptoms begin? Sci Transl Med. 2014;6:228fs13.

2. Farrell ME, Kennedy KM, Rodrigue KM, et al. Association of longitudinal cognitive decline with amyloid burden in middle-aged and older adults: evidence for a dose-response relationship. JAMA Neurol. 2017;74:830-838.
3. Gonneaud J, Arenaza-Urquijo EM, Mezenge F, et al. Increased florbetapir binding in the temporal neocortex from age 20 to 60 years. Neurology. 2017;89:24382446.

4. Grothe MJ, Barthel H, Sepulcre J, Dyrba M, Sabri O, Teipel SJ. In vivo staging of regional amyloid deposition. Neurology. 2017;89:2031-2038.

5. Landau SM, Horng A, Jagust WJ. Alzheimer's disease neuroimaging. I. Memory decline accompanies subthreshold amyloid accumulation. Neurology. 2018;90: e1452-e1460.

6. Leal SL, Landau SM, Bell RK, Jagust WJ. Hippocampal activation is associated with longitudinal amyloid accumulation and cognitive decline. eLife. 2017;6:e22978.

7. Minoshima S. Establishing amyloid PET imaging biomarkers: ongoing efforts. AJNR. 2015;36:1245-1246.

8. Shokouhi S, McKay JW, Baker SL, et al. Reference tissue normalization in longitudinal ${ }^{18} \mathrm{~F}$-florbetapir positron emission tomography of late mild cognitive impairment. Alzheimers Res Ther. 2016;8:2.

9. Landau SM, Fero A, Baker SL, et al. Measurement of longitudinal beta-amyloid change with ${ }^{18} \mathrm{~F}$-florbetapir PET and standardized uptake value ratios. $\mathrm{J} \mathrm{Nucl}$ Med. 2015;56:567-574.

10. Brendel M, Hogenauer M, Delker A, et al. Improved longitudinal $\left[{ }^{18} \mathrm{~F}\right]-\mathrm{AV} 45$ amyloid PET by white matter reference and VOI-based partial volume effect correction. Neuroimage. 2015;108:450-459.

11. Chen K, Roontiva A, Thiyyagura P, et al. Improved power for characterizing longitudinal amyloid-beta PET changes and evaluating amyloid-modifying treatments with a cerebral white matter reference region. J Nucl Med. 2015;56: $560-566$.

12. Schwarz CG, Senjem ML, Gunter JL, et al. Optimizing PiB-PET SUVR changeover-time measurement by a large-scale analysis of longitudinal reliability, plausibility, separability, and correlation with MMSE. Neuroimage. 2017;144: $113-127$.

13. Roher AE, Kuo YM, Esh C, et al. Cortical and leptomeningeal cerebrovascular amyloid and white matter pathology in Alzheimer's disease. Mol Med. 2003;9: $112-122$.

14. Thal DR, Rub U, Orantes M, Braak H. Phases of A beta-deposition in the human brain and its relevance for the development of AD. Neurology. 2002;58:1791-1800.

15. Fodero-Tavoletti MT, Rowe CC, McLean CA, et al. Characterization of PiB binding to white matter in Alzheimer disease and other dementias. J Nucl Med. 2009;50:198-204.

16. Klunk WE, Lopresti BJ, Ikonomovic MD, et al. Binding of the positron emission tomography tracer Pittsburgh compound-B reflects the amount of amyloid-beta in Alzheimer's disease brain but not in transgenic mouse brain. $J$ Neurosci. 2005;25:10598-10606.

17. Harauz G, Ishiyama N, Hill CM, Bates IR, Libich DS, Fares C. Myelin basic protein-diverse conformational states of an intrinsically unstructured protein and its roles in myelin assembly and multiple sclerosis. Micron. 2004;35:503-542.

18. Glodzik L, Rusinek H, Li J, et al. Reduced retention of Pittsburgh compound B in white matter lesions. Eur J Nucl Med Mol Imaging. 2015;42:97-102.

19. Stankoff B, Freeman L, Aigrot MS, et al. Imaging central nervous system myelin by positron emission tomography in multiple sclerosis using [methyl-(1)(1)C]-2(4'-methylaminophenyl)- 6-hydroxybenzothiazole. Ann Neurol. 2011;69:673-680.

20. Lowe VJ, Lundt ES, Senjem ML, et al. White matter reference region in PET studies of ${ }^{11} \mathrm{C}$-Pittsburgh Compound B uptake: effects of age and amyloid- $\beta$ deposition. J Nucl Med. 2018;59:1583-1589.

21. Lois C, Gonzalez I, Johnson KA, Price JC. PET imaging of tau protein targets: a methodology perspective. Brain Imaging Behav. March 1, 2018 [Epub ahead of print]. 